\title{
Constraining Type Ia Supernova Progenitors
}

\author{
E. Scannapieco ${ }^{1}$, C. Raskin ${ }^{1}$, M. Della Valle ${ }^{2}$, C. Fryer ${ }^{3}$, J. Rhoads ${ }^{1}$, \\ G. Rockefeller ${ }^{3}$, and F. X. Timmes ${ }^{1}$ \\ ${ }^{1}$ School of Earth and Space Exploration, Arizona State University, Tempe, AZ, USA \\ ${ }^{2}$ INAF- Osservatorio Astronomico di Capodimonte, Salita Moiariello, 16-80131, Napoli, Italy \\ ${ }^{3}$ CCS-2, Los Alamos National Laboratories, Los Alamos, NM, USA
}

\begin{abstract}
We present observational and theoretical studies constraining Type Ia supernova progenitors.

First, we use a new observational technique to show that "prompt" SNe Ia that trace starformation on cosmic timescales exhibit a significant delay time of 200-500 million years. This implies that either the majority of SNe Ia companion stars have main-sequence masses less than three solar masses, or that most SNe Ia arise from double-white dwarf binaries.

Second we present a comprehensive study of white dwarf collisions as an avenue for creating SNe Ia. Using a smooth particle hydrodynamics code with a 13-isotope nuclear network, we show that several combinations of white dwarf masses and impact parameters produce enough ${ }^{56} \mathrm{Ni}$ to result in luminosities ranging from those of sub-luminous to super-luminous SNe Ia, depending on the parameters of the collision.

Finally, we conduct a simulation survey of double-degenerate white dwarf mergers with varying mass combinations. Unlike previous works, we do not add detonations by hand to our simulations, and we do not find any thermonuclear explosions during the mergers. Instead, all but one of our simulations forms a cold, degenerate core surrounded by a hot disk, while our least massive pair of stars forms only a hot disk. We characterize the remnants by core mass, rotational velocity, and half-mass radius, and discuss how we will evolve them further with simulations that incorporate dissipative processes. Such simulations may indeed lead to double-degenerate Type Ia explosions that occur many orbits after the mergers themselves.
\end{abstract}

Keywords. supernovae

\section{Constraining the Type Ia Time Delay}

Type Ia supernovae (SNe Ia) serve as the primary cosmological standard candles; however, their regularity need not imply that they share a common progenitor. Indeed, one striking bimodality is that the brightest SNe Ia occur in star-forming galaxies, and the dimmest occur in galaxies with little star-formation (e.g. van den Bergh \& Filippenko 2005). In fact, while several parameterizations of the SNe Ia rate exist (e.g. Kobayashi et al. 2000; Greggio 2005), current data is well fit by a two-component parameterization (Mannucci et al. 2005; Scannapieco \& Bildsten 2005), which takes the form $S N R_{\mathrm{Ia}}(t)=$ $A M_{*}(t)+B \dot{M}_{*}(t)$. Here, we refer to the A-component, which is proportional to the total stellar mass of the host, as "delayed," and to the B-component, which is proportional to the instantaneous star-formation rate (e.g. Oemler \& Tinsley 1979), as "prompt." However, the prompt component can not follow the instantaneous star-formation rate since SNe Ia can not arise before stars evolve to form white-dwarfs and before these white dwarfs accrete mass from their companions. Thus, it is likely that the prompt component exhibits its own delay time, $\tau$, such that $S N R_{\text {Ia }}(t)=A M_{*}(t)+B \dot{M}_{*}(t-\tau)$.

To measure this delay we developed a new method based on the approach in Fruchter et al. (2006). Here, for each host galaxy, the authors computed the fraction of the total 
light in all pixels fainter than the pixel containing a transient. By compiling such measurements into a cumulative histogram, they demonstrated that long-duration gamma-ray bursts are more likely than core-collapse supernovae (SNcc) to cluster in the brighter regions of a galaxy. Kelly et al. (2008) expanded upon this analysis to distinguish between SNcc subtypes Ic, Ib, and type II. However, this study showed no difference between SNcc and SNe Ia, because the radial gradient in the density of stars in the host galaxies obscures the effect of a time delay.

We call our solution to this problem the doughnut method (Raskin et al. 2009a). The idea is to use the light within \pm 0.5 radial scale length of the supernova: wide enough to encompass a good representative sample, yet narrow enough to represent local variations in the host. Applying this methods to a sample drawn from the Padova-Asiago Supernova Catalogue resulted in 50 usable Sloan Digital Sky Survey (SDSS) $g$-band images, and as a control sample, 74 images of SNcc hosts. Our results appear in Figure 1.

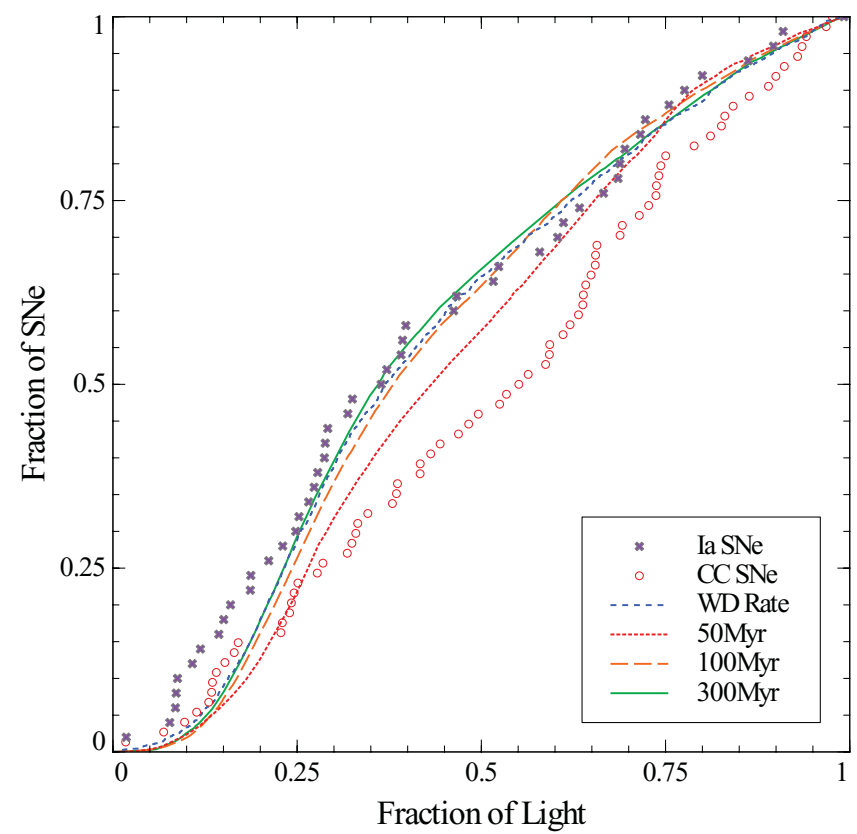

Figure 1. A histogram of the number of SNe Ia (crosses) located in pixels brighter than a given fraction of the $g$-band light contained within a surrounding "doughnut," which shows that SNe Ia tend to occur in dimmer regions. A histogram of SNcc (circles) is also shown, which differs at the $99.6 \%$ confidence level. Finally, curves for the A+B model for several delay times as well as the white dwarf formation rate model are shown. Both the $\tau=300$ and a model that traces the white dwarf formation rate provide excellent fits to the data. (From Raskin et al. 2009a)

Unlike analyses that use the full galaxy light, our approach is able to distinguish between the SNcc and SNe Ia distributions with $99.6 \%$ confidence, as quantified by a Kolmogorov-Smirnov (KS) test. SNe Ia are clearly biased to fainter pixels, while the SNcc distribution closely follows the $g$-band light distribution. By comparing our observations to analytic models (Raskin et al. 2008), we establish a strong case for a modified $\mathrm{A}+\mathrm{B}$ model in which the prompt component is delayed by 300-500 Myrs. Note that this is an average time and that there can be a considerable spread in this value. Alternatively, we find that a model in which the SNe Ia rate is directly proportional to the white-dwarf formation rate (Pritchet et al. 2008) reproduces the observed spatial distributions of SNe Ia very well. Regardless of which model proves to be the best fit, these results imply 
that that either the majority of Ia companion stars have main-sequence masses less than three solar masses, or that most SNe Ia arise from double-white dwarf binaries.

\section{Double Degenerate Collisions}

These results suggest that double-degenerate binaries may contribute to the SNeIa rate, and that such scenarios merit further investigation. To this end, we used a version of the 3D Smoothed Particle Hydrodynamics code SNSPH (Fryer et al. 2006) to which we added a 13 isotope $\alpha$-chain nuclear reaction network and a Helmholtz free energy based equation of state as described in (Raskin et al. 2009b). The first scenario we explored was that of white dwarf collisions, which occur in dense environments such as globular cluster cores (Raskin et al. 2010). The initial conditions for white dwarfs were generated using a fourth-order solver that integrated the kinematic equations. The impactor star was given a small initial velocity comparable to the velocity dispersion of globular cluster cores, $\sigma=10 \mathrm{~km} / \mathrm{s}$. The results of one such collision, two $0.64 \mathrm{M}_{\odot}$ white dwarfs colliding with an impact parameter of $b=1$, is shown in the left panels of Figure 2.

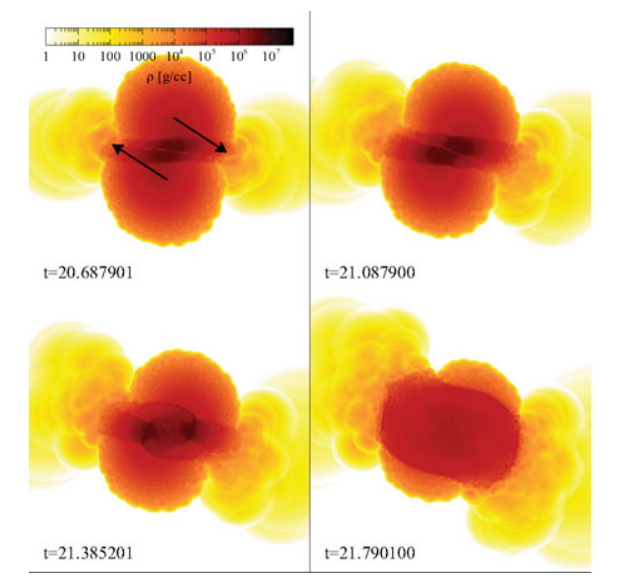

\begin{tabular}{c|cc|c|ccc}
\hline \hline$\#$ & $m_{1}\left[\mathrm{M}_{\odot}\right]$ & $m_{2}\left[\mathrm{M}_{\odot}\right]$ & $m_{\text {tot }}\left[\mathrm{M}_{\odot}\right]$ & $b=0$ & $b=1$ & $b=2$ \\
\hline 1 & 0.64 & 0.64 & 1.28 & 0.51 & 0.47 & $\not{a}$ \\
2 & 0.64 & 0.81 & $\mathbf{1 . 4 5}$ & 0.14 & 0.53 & $\not{a}$ \\
3 & 0.64 & 1.06 & $\mathbf{1 . 7 0}$ & 0.26 & $\not{a}$ & $\not{a}$ \\
4 & 0.81 & 0.81 & $\mathbf{1 . 6 2}$ & 0.84 & 0.84 & 0.65 \\
5 & 0.81 & 1.06 & $\mathbf{1 . 8 7}$ & 0.90 & 1.13 & $\not{a}$ \\
6 & 0.96 & 0.96 & $\mathbf{1 . 9 2}$ & 1.27 & 1.32 & 1.33 \\
7 & 1.06 & 1.06 & $\mathbf{2 . 1 2}$ & $\mathbf{1 . 7 1}$ & $\mathbf{1 . 7 2}$ & $\mathbf{1 . 6 1}$ \\
8 & 0.50 & 0.50 & 1.00 & 0.00 & - & - \\
\hline
\end{tabular}

Figure 2. Left: A $2 \mathrm{D}$ slice of densities through the $x-y$ plane of the $b=1$ case of two $0.64 \mathrm{M}_{\odot}$ white dwarfs colliding. Four snapshots at different times are shown. Arrows in the top-left panel indicate the direction of motion of each star. Right: Simulation of ${ }^{56} \mathrm{Ni}$ yields for various mass combinations and impact parameters ( $b$, the vertical separation between the cores of both white dwarfs at the moment of impact in units of the radius of the primary white dwarf). Values in bold are super- Chandrasekhar masses, and values indicated with a (@) are those simulations that resulted in remnants. Dashes (-) indicate combinations of parameters we did not simulate. (From Raskin et al. 2010.)

When the stars first collide, the infall speed of the material entering the shocked region is greater than the sound speed, resulting in a stalled shocks. Less than two seconds after the stars first collide, sufficiently high temperatures and densities are reached at the edges of the shocked region to initiate detonations. The sound speeds in these zones are raised higher than the infall speeds due to the rapid rise in temperature, ${ }^{56} \mathrm{Ni}$ begins to appear in large quantities, and the stars are disrupted.

A table of results from a suite of similar simulations appears in the right panel of Figure 2. In more than $75 \%$ of the cases, a significant mass of ${ }^{56} \mathrm{Ni}$ was produced. Even mass pairs below the Chandrasekhar limit exhibited explosive nuclear burning, with the $0.64 \mathrm{M}_{\odot} \times 2$ mass pair producing ${ }^{56} \mathrm{Ni}$ in quantities comparable to standard SNe Ia. Moreover, regardless of the impact parameter, the most massive pairs of stars produced 
quantities of ${ }^{56} \mathrm{Ni}$ consistent with those of SNe Ia with super-Chandrasekhar mass progenitors.

\section{Double Degenerate Mergers}

On the other hand, as the rate of such collisions is likely to be $10-100$ per year within $z<1.0$, they can not make up the majority of observed SNe Ia. A much more common double degenerate case involves the merger of two white dwarfs in a tight binary (e.g. Iben \& Tutukov 1984; Benz et al. 1989). Using the same code as in our collision simulations, we simulated a large set of tidally locked white dwarfs beginning from a metastable configuration in which the stars were still in hydrostatic equilibrium but tidally distorted (e.g. Rosswog et al. 2010). Unlike Pakmor et al. (2010) we did not insert detonations by hand at the location at which large-scale conditions matched those found in offline calculations to produce the microphysical conditions necessary for detonation.
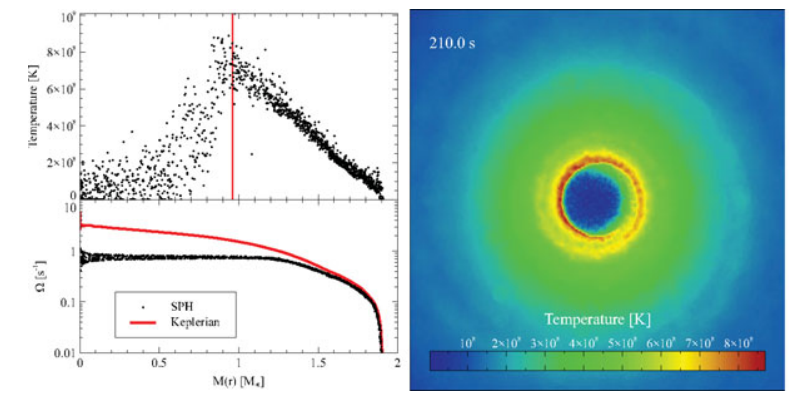

\begin{tabular}{c|cc|cccc}
\hline \hline$\#$ & $m_{1}$ & $m_{2}$ & $M_{\text {disk }}$ & $M_{\text {core }}$ & $r_{d}$ & $\Omega\left[\mathrm{s}^{-1}\right]$ \\
\hline 1 & 0.64 & 0.64 & 0.44 & $0.84-0.99$ & 0.019 & 0.36 \\
2 & 0.64 & 0.81 & 0.49 & $0.96-1.05$ & 0.023 & 0.38 \\
3 & 0.64 & 0.96 & 0.53 & $1.07-1.16$ & 0.028 & 0.45 \\
4 & 0.64 & 1.06 & 0.59 & $1.11-1.20$ & 0.029 & 0.46 \\
5 & 0.81 & 0.81 & 0.57 & $1.05-1.20$ & 0.019 & 0.47 \\
6 & 0.81 & 0.96 & 0.67 & $1.10-1.26$ & 0.020 & 0.57 \\
7 & 0.81 & 1.06 & 0.75 & $1.12-1.30$ & 0.019 & 0.69 \\
8 & 0.96 & 0.96 & 0.67 & $1.25-1.44$ & 0.016 & 0.67 \\
9 & 0.96 & 1.06 & 0.79 & $1.23-1.42$ & 0.017 & 0.78 \\
10 & 1.06 & 1.06 & 0.87 & $1.25-1.48$ & 0.016 & 0.78 \\
\hline
\end{tabular}

Figure 3. Left: Temperature and angular velocity as functions of mass coordinate for the remnant configuration of our $0.96 \mathrm{M}_{\odot} \times 2$ simulation at $210.0 \mathrm{~s}$ after the destruction of the secondary. The red vertical line indicates the progenitor mass of the primary star. Center: A slice in the $\mathrm{x}-\mathrm{y}$ plane of the temperature of the remnant in this simulation. Right: Simulated binary mass pairs and their disk properties. The half-mass radius of the disk is given as $r_{d} . \Omega$ is the rotational speed of the inner disk. The core mass, $M_{\text {core }}$, is quoted with the upper limit representing the mass that the core would grow to if thermal support was removed from the disk. All units are solar unless specified otherwise. (From Raskin et al. 2011)

In fact, no detonations were seen in our simulations. Instead all but one pair of stars formed a cold, degenerate core surrounded by a hot disk, as shown in the left panels of Figure 3 while our least massive pair formed only a hot disk. We characterize our remnant configurations by the core mass, rotational velocity of the core, in the right panel of Figure 3. Since the disks are optically thick, the surface layers will lose energy through radiation, and the material will sink to lower latitudes, driving convective currents. While not yet included in our simulations, we expect this convection will mimic an $\alpha$-disk prescription, and thus, the accretion times will be on the order of a few minutes. Furthermore, our three most massive stellar pairs lead to configurations whose cores might grow to super-Chandrasekhar masses on even shorter timescales, after cooling has removed some of the thermal support from the inner portions of the surrounding disks.

This work was supported by NSF grant AST08-06720 and NASA NESSF grant PVS0401.

\section{References}

Benz, W., Cameron, A. G. W., \& Bowers, R. L. 1989 in G. Wegner (eds.), in White Dwarfs, Proc. IAU Symposium No. 114 (Berlin: Springer-Verlag), p. 511 
Fryer, C. L., Rockefeller, G., \& Warren, M. S. 2006, ApJ, 643, 292

Fruchter, A. S., et al. 2006, Nature, 441, 463 L

Greggio, L. 2005, A\&\&A, 441, 1055

Iben, Jr., I. \& Tutukov, A. V. 1984, ApJS, 54, 335

Kelly, P. L., Kirshner, R. P., \& Pahre, M. 2008, ApJ, 687, 1201

Kobayashi, C., Tsujimoto, T. \& Nomoto, K. 2000, ApJ, 539, 26

Mannucci, F., Della Valle, M. \& Panagia, N. 2006, MNRAS, 370, 773

Oemler \& Tinsley 1979, AJ, 84, 985

Pakmor, R., et al. 2010, Nature, 463, 61

Pritchet, C. J., Howell, D. A., \& Sullivan, M. 2008, ApJ, 683, L25

Raskin, C., Scannapieco, E., Rhoads, J., \& Della Valle, M 2008, ApJ, 689, 358

Raskin, C., Scannapieco, E., Rhoads, J., \& Della Valle, M 2009a, ApJ, 707, 74

Raskin, C., Timmes, F.X., Scannapieco, E., Diehl, S., \& Fryer, C. 2009b, MNRAS, 399, 156

Raskin, C., et al. 2010, ApJ, 724, 111

Raskin, C., Scannapieco, E., Rockefeller, G., Fryer, C., \& Timmes, F.X. 2011, ApJ, submitted

Rosswog, S., Kasen, D., Guillochon, J., \& Ramirez-Ruiz, E. 2009 ApJ, 705, L128

Scannapieco, E. \& Bildsten, L. 2005, ApJ, 629, L85

van den Bergh, S., Li, W. \& Filippenko, A. V. 2005, PASP, 834, 773 